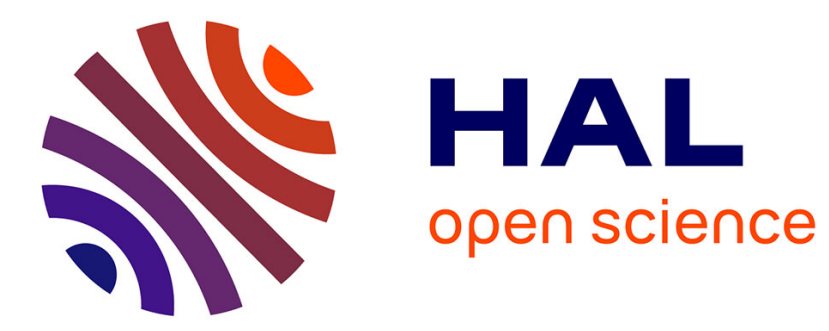

\title{
A procedure for identifying the plastic behaviour of single crystals from the local response of polycrystals
}

Thierry Hoc, Jérôme Crépin, Lionel Gélébart, André Zaoui

\section{To cite this version:}

Thierry Hoc, Jérôme Crépin, Lionel Gélébart, André Zaoui. A procedure for identifying the plastic behaviour of single crystals from the local response of polycrystals. Acta Materialia, 2003, 51, pp.54795490. 10.1016/S1359-6454(03)00413-0 . hal-00111378

\section{HAL Id: hal-00111378 \\ https://hal.science/hal-00111378}

Submitted on 13 Aug 2019

HAL is a multi-disciplinary open access archive for the deposit and dissemination of scientific research documents, whether they are published or not. The documents may come from teaching and research institutions in France or abroad, or from public or private research centers.
L'archive ouverte pluridisciplinaire HAL, est destinée au dépôt et à la diffusion de documents scientifiques de niveau recherche, publiés ou non, émanant des établissements d'enseignement et de recherche français ou étrangers, des laboratoires publics ou privés.

\section{다)(1) $(5$}

Distributed under a Creative Commons Attribution - NonCommerciall 4.0 International 


\title{
A procedure for identifying the plastic behavior of single crystals from the local response of polycrystals
}

\author{
T. Hoc ${ }^{\mathrm{a}, *}$, J. Crépin ${ }^{\mathrm{b}}$, L. Gélébart ${ }^{\mathrm{b}}$, A. Zaoui ${ }^{\mathrm{b}}$ \\ a MSSMAT, UMR 8579, Ecole Centrale Paris, Grande voie des vignes, 92295 Châtenay Malabry, France \\ b LMS, CNRS_Ecole Polytechnique, 91128 Palaiseau, France
}

The overall and local tensile responses of an $\alpha$ iron multicrystalline sample are investigated in order to derive the plastic constitutive equations for the constituent single crystals. The macroscopic stress-strain curve and some statistical characteristics of the strain field measured on the sample surface are compared with their simulated counterparts. The optimal values of the material parameters of four types of hardening laws are derived by a minimization procedure. The best results are obtained with a nonlinear anisotropic law which uses the dislocation densities on the slip systems. This procedure is then validated on a fine-grained polycrystalline sample of a similar material by using the measured displacement field on the edge of a selected area as boundary conditions for finite element method (FEM) computation. The resulting optimal material parameters for the single crystal are found to be consistent with the values available in the literature, and the whole simulated strain fields as well as the evolution of the crystallographic texture, is compared satisfactorily with the experimental data.

Keywords: Steels; Plastic deformation; Scanning electron microscope (SEM); Finite element analysis

\section{Introduction}

In the last few decades, important advances have been made in developing efficient micromechanical models for the prediction of the overall plastic and viscoplastic behavior of polycrystalline materials from that of the constituent crystals. Nevertheless, several difficulties still prevent fully

* Corresponding author. Tel.: +33-1-41-13-16-16; fax: +331-41-13-14-30.

E-mail address: hoc@mssmat.ecp.fr (T. Hoc). guaranteed and convenient prediction tools, with a wide validity range, from being available for industrial needs in the field of metal forming. Two main problems can be considered as being among the most critical ones.

On one hand, the improved polycrystalline models which have been derived recently from powerful nonlinear homogenization techniques (e.g. [13]) lead to reduced intergranular interactions and softer overall responses with respect to previous ones of the Taylor [4] or Hill-Hutchinson [5,6] type. As a consequence, it becomes even more necessary to reach an accurate description of the 
plastic or viscoplastic constitutive behavior of the constituent grains. This can be performed through direct mechanical investigations on single crystals but, in addition to needing demanding and expensive experimental tests, such investigations cannot yield definitive answers to the questions addressed. Crystals and polycrystals with the same chemical composition can hardly be tested and only simple homogeneous mechanical tests can generally be performed.

On the other hand, many important mechanical properties of polycrystals cannot be deduced solely through direct upscaling from single crystal plasticity. Damage, as well as phase transformations, recrystallization, shear banding, etc., depend strongly on very local mechanical states that are beyond the scope of homogenization procedures, since the latter hardly give access to more than average values. Similarly, the determination of critical values for the activation of various mechanisms (slip, twinning, cleavage, debonding, etc.) and the analyses of strain heterogeneity or strain localization cannot be done unambiguously with tests on single crystals. Furthermore, specific strain heterogeneity patterns may develop in polycrystals in the early plastic regime, as observed under uniaxial tension in steel [7] or zirconium alloys [8], which consist of strain bands oriented at $\pm 45^{\circ}$ to the tensile axis. Such bands extend over several grains and obviously cannot be predicted by mean field polycrystalline models.

Fortunately, two types of approaches have been developed intensively recently, which provide new tools for addressing these difficult problems: experimental microextensometry techniques and advanced numerical simulations. For the last two decades, the development of microextensometric techniques [9-11], combined with image correlation analysis, has made it possible to measure accurate displacement and strain fields on the surface of samples. In particular, the use of scanning electron microscopes (SEMs) now allows characterization of the deformation of large areas, several square millimeters, in relation with the microstructure. In parallel, the electron back-scattering diffraction (EBSD) techniques give access to the lattice orientation of the individual grains, whereas the orientation imaging microscopy (OIM) tech- nique, which is based on the EBSD scan, makes it possible to combine microextensometry measurements with the determination of the local lattice orientation field evolution. On the other hand, the amazing increase in numerical computation capabilities has made it possible to simulate large polycrystalline areas and to derive local mechanical fields by the finite element method (FEM) [11-14].

Consequently, significant comparisons can now be performed between accurate strain field measurements and their simulated counterparts. Of course, the constitutive equations which are used in the finite element simulations cannot be taken for granted, since they are generally what one is looking for. In the first approximation, however, they may be improved from this comparison itself so as to lead to improved physically based constitutive equations $[15,16]$. To the best of our knowledge, the first studies that compared such experimental data and numerical simulations were published by Becker and Panchanadeeswaran [17] in 1995. These results showed good agreement for some grains and not such a good agreement for others. Further, similar studies performed on multicrystals [18] and polycrystals [19] in order to predict their strain heterogeneity, although qualitatively interesting, predicted a degree of heterogeneity somewhat smaller than that recorded experimentally. This can be due to the fact that the material parameters used in the crystal constitutive equations were derived from single crystal investigations $[20,21]$ or from indirect identification. This reverse analysis used macroscopic experimental responses and an upscaling polycrystal model [22]. In order to improve the accuracy of the mechanical prediction, a few recent studies [11,23,24] also used measured local strain fields.

This paper aims at reporting a novel procedure for identifying the material parameters of single crystals in the plastic regime by comparing the experimental response of a polycrystalline sample to FEM computations. This response does not reduce to the macroscopic tensile stress-strain curve, but also includes some statistical characteristics of the strain field within the grains. The first sample studied is a multicrystal with only one grain through the thickness and a quasi-2D microstruc- 
ture (Section 2). Two kinds of constitutive equations have been checked. The simple ones use the slip rates on the easy glide slip systems as hardening parameters and the more complex ones are based on the dislocation densities on the slip systems. The proposed procedure, which takes recourse to the statistical strain distribution at several prescribed strain levels, definitely shows the merits of the latter description with respect to the former one, especially when anisotropic hardening interactions are taken into account. These results are validated on a second fine-grained polycrystalline sample constituted with a similar material (Section 3). The same experimental techniques are used for straining and characterizing this sample and the FEM computation makes use of constitutive equations derived from the same identification procedure. The boundary conditions are, however, no more homogeneous, but defined by the measured displacement field on the edge of a given area of the sample. The comparison of the measured and computed strain fields in this area, as well as that of the overall stress-strain responses and of the crystallographic texture evolutions, shows fairly good agreement. This illustrates the advantages of the present novel identification procedure as compared with former ones. Finally, the physical soundness of the values found for the material parameters of the selected constitutive law is discussed briefly and justified in physical terms (Section 4).

\section{Identification procedure}

\subsection{Material and experimental techniques}

The selected material is an ARMCO iron multicrystal whose chemical composition is described in Table 1.

The ARMCO multicrystal, of dimensions
$16 \times 4 \mathrm{~mm}^{2}$, is fitted with an extensometer. This sample has only one grain through the thickness and the grains have a diameter of $2 \mathrm{~mm}$ along the large faces. The tensile test is performed along the one axis in the chamber of a SEM. The local strain field on the sample surface is measured by a microextensometry technique $[8,25]$, whose principle consists in taking SEM images of a square microgrid deposited on the surface before and after deformation. The in-plane displacements of the crosses of this microgrid are determined by a correlation method. The strain field is then derived from this displacement field. The choice of the pitch of the microgrids depends on the precision and resolution required to measure intragranular strain fields. For the multicrystal, it can be fixed at $20 \mu \mathrm{m}$, whereas it has to be about 10 times smaller for the polycrystalline sample (Section 3 ). The lattice orientation of each grain is measured by EBSD. The whole orientation field associated with the studied microstructure is obtained by automatically prescribing the electron beam displacement for steps of $1 \mu \mathrm{m}$ on a regular mesh.

\subsection{Experimental results}

Fig. 1 shows the initial crystallographic orientation field for the multicrystal. All the 27 grains along the gauge length of the sample have been indexed. This field results from about 160000 measurements at all the points of the fiducial grids, i.e., it includes 5000 points per grain.

In Fig. 2, typical measurements of the axial strain field over the multicrystalline sample are reported for a prescribed overall axial strain of $5 \%$.

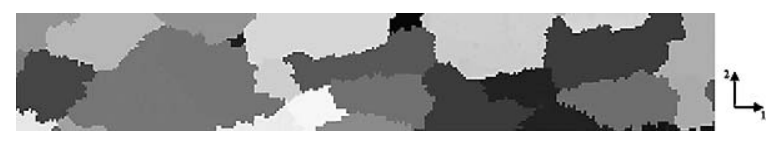

Fig. 1. Crystallographic orientation field for the multicrystal.

Table 1

Chemical composition of the multicrystalline sample $\left(10^{-3}\right.$ wt.\%)

\begin{tabular}{llllllllllll}
\hline & $\mathrm{C}$ & $\mathrm{Mn}$ & $\mathrm{P}$ & $\mathrm{S}$ & $\mathrm{Al}$ & $\mathrm{Ti}$ & $\mathrm{Si}$ & $\mathrm{Cu}$ & $\mathrm{Cr}$ & $\mathrm{Ni}$ & $\mathrm{Fe}$ \\
\hline $\mathrm{ARMCO}$ & 4 & $<5$ & 8 & 2 & $<5$ & - & $<5$ & $<5$ & $<5$ & - & $\mathrm{Bal}$ \\
\hline
\end{tabular}



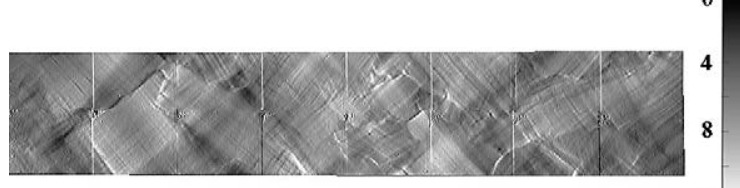

Fig. 2. Experimental $E_{11}$ axial strain field for the multicrystalline sample.

These results are in qualitative agreement with literature results [18]. In particular, the maximum value of the local axial strain is about two times larger than the macroscopic one. The strongest strain values are frequently localized near the grain boundaries but they seem to draw an overall pattern inclined at $\pm 45^{\circ}$ to the tensile axis.

In view of the subsequent identification procedure, these local strain heterogeneities, normalized by the macroscopic axial strain, are characterized by their statistical distribution (Fig. $3)$.

This information is richer than the set of strain averages per grain; it will be taken in what follows as the main experimental basis for comparison with simulated results, together with the overall stressstrain response.

\subsection{Plasticity model for the single crystal}

\subsubsection{Framework}

A crystalline plasticity constitutive model, which accounts for crystallographic glide and lat-

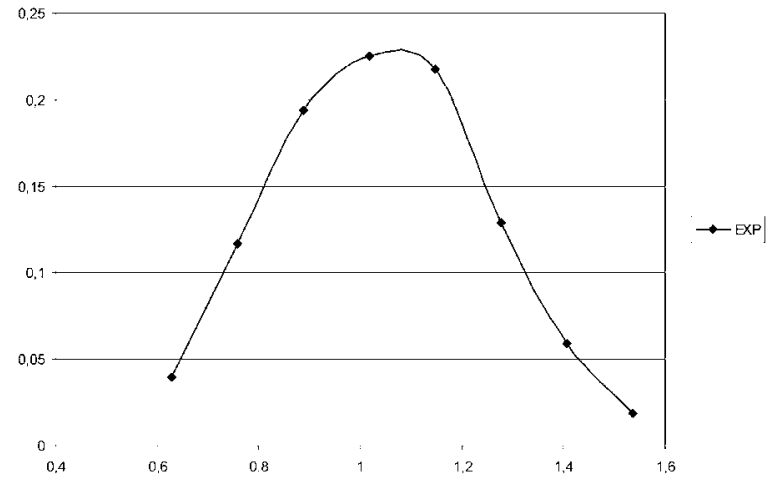

Fig. 3. Statistical distribution of the normalized local strain $\left(E_{11}\right)$ values for the multicrystalline sample (5\% macroscopic strain). tice rotation at finite strain, has been implemented in the ABAQUS FE code. Such an implementation of the constitutive relations as a "User Material" subroutine in the ABAQUS code has been described by Smelser and Becker [26,27]. The mechanical modeling is consistent with the framework developed by Peirce et al. [28] and also adopted by Teodosiu et al. [15]. The kinetics of large transformations is based upon the multiplicative decomposition of the deformation gradient into its elastic and plastic parts. The velocity gradient $\underline{\mathbf{L}}$ is decomposed additively into an elastic part $\underline{\mathbf{L}}^{\mathrm{e}}$ and a plastic part $\underline{\mathbf{L}}^{\mathrm{p}}$ due to glide on crystallographic slip systems. A system $(s)$ is defined by its unit slip direction $\overrightarrow{\mathbf{g}}^{(s)}$ and unit slip plane normal $\overrightarrow{\mathbf{n}}^{(s)}$ :

$\underline{\mathbf{L}}=\underline{\mathbf{D}}+\underline{\mathbf{W}}=\underline{\mathbf{L}}^{\mathrm{e}}+\sum_{s} \dot{\gamma}^{(s)} \overrightarrow{\mathbf{g}}^{(s)} \otimes \overrightarrow{\mathbf{n}}^{(s)}$

where $\underline{\mathbf{D}}$ and $\underline{\mathbf{W}}$ are the symmetric and antisymmetric parts of $\underline{\mathbf{L}}$, respectively and $\dot{\gamma}^{(s)}$ is the slip rate on system $(s)$. The Jaumann derivative of the Kirchhoff stress tensor $\stackrel{\nabla}{\boldsymbol{\tau}}$ is associated to the elastic strain rate through the elastic moduli $\underline{\underline{\mathbf{K}}}$, which are the usual elastic moduli when the elastic strains are small. $\underline{\boldsymbol{\tau}}$ is given by

$\underline{\nabla}=\underline{\underline{\mathbf{K}}}: \underline{\mathbf{D}}-\sum_{s} \dot{\boldsymbol{\gamma}}^{(\mathrm{s})} \underline{\mathbf{R}}^{(\mathrm{s})}$

$\underline{\mathbf{R}}^{(s)}$ is a tensor describing the slip geometry. It can be expressed as

$\underline{\mathbf{R}}^{(\mathrm{s})}=\underline{\mathbf{K}}: \underline{\mathbf{P}}^{(\mathrm{s})}+\underline{\mathbf{W}}^{(\mathrm{s})} \cdot \underline{\boldsymbol{\tau}}-\underline{\boldsymbol{\tau}} \cdot \underline{\mathbf{W}}^{(\mathrm{s})}$

where $\underline{\mathbf{P}}^{(s)}$ is the symmetric part of $\overrightarrow{\mathbf{g}}^{(s)} \otimes \overrightarrow{\mathbf{n}}^{(s)}$ and $\underline{\mathbf{W}}^{(s)}$ its antisymmetric part.

The plastic slip rates obey a viscoplastic power law with a threshold value. The activation of a slip system $(s)$ is governed by Schmid's law, i.e., $\left|\tau^{(s)}\right| \geq \tau_{\mathrm{c}}^{(s)}$, where $\tau^{(s)}$ is the resolved shear stress on slip system $(s)$ and $\tau_{\mathbf{c}}^{(s)}$ the associated critical shear stress, which depends on the structural variables and temperature:

$\dot{\gamma}^{(s)}=\dot{\gamma}_{\mathrm{o}}\left|\frac{\tau^{(s)}}{\tau_{\mathrm{c}}^{(s)}}\right|^{n} \operatorname{sgn}\left(\tau^{(s)}\right)$ if $\left|\tau^{(s)}\right| \geq \tau_{\mathrm{c}}^{(s)}$

and $\dot{\gamma}^{(s)}=0$ otherwise 
Here, $\dot{\gamma}_{\mathrm{o}}$ is a reference strain rate which is usually of the order of $10^{-3}$ at room temperature for metallic materials, while the exponent $n$ is of the order of 100 .

\subsubsection{The case of bcc crystals}

For body-centered cubic (bcc) crystals, such as $\alpha$-iron, glide occurs along the $<111>$ directions and can be considered as operating on the $\left\{\begin{array}{lll}1 & 1 & 0\end{array}\right\}$ and $\left\{\begin{array}{lll}1 & 1 & 2\end{array}\right\}$ planes, which leads to $N_{\text {sys }}=24$ slip systems [29,30]. The hardening characteristics depend on the interaction mechanisms between the different (activated and latent) slip systems. They are commonly modeled by a hardening matrix $\mathbf{h}$ defined by

$$
\dot{\tau}_{\mathrm{c}}^{(s)}=\sum_{u} h^{s u} \dot{\gamma}(u)
$$

For the sake of simplicity, the hardening matrix coefficients can be taken to be constant with only two values, one for the diagonal terms, expressing self-hardening, and another one for the off-diagonal terms, which is associated with latent hardening. This linear hardening description (A and B types, with two and three parameters, respectively, according to whether the $\mathbf{h}$ matrix is isotropic or not) is adopted as a first approximation in what follows (see Table 2).

For a detailed description, the coefficients of the hardening matrix can be expressed in terms of internal variables whose evolution is related to the deformation history [31]. Here, these internal variables are chosen as the average dislocation densities $\rho^{(s)}$ on each slip system $(s)$. The evolution of the total dislocation density, based on Orowan's relation and an annihilation process of dislocation dipoles [32], is given by
$\dot{\rho}^{(s)}=\frac{1}{b}\left(\frac{1}{L^{(s)}}-G_{\mathrm{c}} \rho^{(s)}\right)\left|\dot{\gamma}^{(s)}\right|$

where $b$ is the magnitude of the Burgers vector, $G_{\mathrm{c}}$ a parameter proportional to the characteristic length associated with the annihilation process of dislocation dipoles and $L^{(s)}$ the mean free path of system $(s)$. The latter can be expressed as

$L^{(s)}=K\left(\sum_{u \neq s} \rho^{(u)}\right)^{-1 / 2}$

where $\rho^{(u)}$ is the total dislocation density on latent systems $(u)$ and $K$ a material parameter. This model is inspired from earlier work by Mecking and Kocks [33] and Teodosiu et al. [15] for fcc crystals and adapted to the bcc structure at intermediate temperatures. The critical resolved shear stress on system $(s)$ is composed of two components $[34,35]$ and can be related to the dislocation densities by:

$\tau_{\mathrm{c}}^{(s)}=\tau_{0}+\mu b \sqrt{\sum_{u} a^{(s)(u)} \rho^{(u)}}$

where the friction stress $\tau_{0}$ is assumed to be the same on all the slip systems and depends on temperature. The interactions between two families of dislocations $(s)$ and $(u)$ are characterized by the matrix $a^{(s)(u)}$, whose terms are taken constant with two values, for the diagonal terms and the off-diagonal terms, respectively.

In what follows, two versions of this nonlinear hardening description are used (cf. Table 2): the isotropic variant ( $\mathrm{C}$ type, five parameters) and the anisotropic variant (D type, six parameters).

Table 2

Parameters for the description of the crystal behavior

\begin{tabular}{|c|c|c|c|c|c|c|}
\hline Linear hardening & $\tau_{\mathrm{c}}^{(s)}(t=0)$ & $h^{s s}$ & $h^{s u}$ & & & \\
\hline A & $\tau_{0}^{\mathrm{A}}$ & $H_{0}^{\mathrm{A}}$ & $H_{0}^{\mathrm{A}}$ & & & \\
\hline B & $\tau_{0}^{\mathrm{B}}$ & $H_{0}^{\mathrm{B}}$ & $q^{\mathrm{B}} H_{0}^{\mathrm{B}}$ & & & \\
\hline Nonlinear hardening & $\tau_{0}$ & $a^{s s}$ & $a^{s u}$ & $\rho_{0}$ & $K$ & $G_{\mathrm{c}}$ \\
\hline $\mathrm{C}$ & $\tau_{0}^{\mathrm{C}}$ & $a_{0}^{\mathrm{C}}$ & $a_{0}^{\mathrm{C}}$ & $\rho_{0}^{\mathrm{C}}$ & $K^{\mathrm{C}}$ & $G_{\mathrm{c}}^{\mathrm{C}}$ \\
\hline D & $\tau_{0}^{\mathrm{D}}$ & $a_{0}^{\mathrm{D}}$ & $q^{\mathrm{D}} a_{0}^{\mathrm{D}}$ & $\rho_{0}^{\mathrm{D}}$ & $K^{\mathrm{D}}$ & $G_{\mathrm{c}}^{\mathrm{D}}$ \\
\hline
\end{tabular}




\subsection{Identification method}

For the four types of constitutive equations considered, the set of optimal material parameters is derived through the automatic software SiDoLo [36]. This procedure consists in a quantitative comparison between experimental and simulated data, with the measured and simulated strain values defined as local averages on the same area. The latter have been obtained by finite element computation of the stress and strain fields of the simulated sample, subjected to the same boundary conditions as the experimental one. The shape and initial lattice orientation of the grains are determined by EBSD measurements (Fig. 4).

Two sets of data are available. The first one is derived from the overall stress-strain response and the second one consists of the reduced statistical distribution of the local strain values. The procedure makes use of both sets, which are combined with a given weight in the global functional to be minimized. This functional integrates, for each set of parameters $A$, the distance between the measured and computed values. It is written in the form:

$\Phi(A)=\sum_{n=1}^{N} \varphi_{n}(A) P_{n}$

with

$\varphi_{n}(A)=\frac{1}{T_{n_{i}}} \sum_{n}^{T_{n}}\left[E c_{n}(i)\right]^{\mathrm{t}}$

Here, $N$ is the total number of values considered $(N=9$ : eight values come from the statistical distribution of the $E_{11}$ strain components and one value is derived from the tensile stress-strain curve). $P_{n}$ is a weighting matrix, which expresses the relative weight one wishes to apply to the various data; $\left[E c_{n}(i)\right]^{\mathrm{t}}$ denotes a transposed matrix formed with the difference between the measured and computed values of the $n$th data taken at "time" $(i) ; T_{n}$ stands

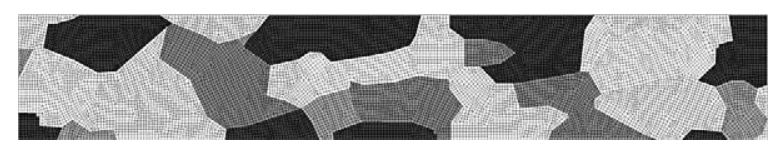

Fig. 4. Meshing of the simulated multicrystal. for the total set of observation "times" for the $n$th data.

As the objective was to compare the local strain fields predicted by several types of constitutive equations, in order to determine which one reproduces the best overall response, a larger weight was attributed to the global stress-strain curve than to the local strain distribution $(0.6$ and 0.1 , respectively).

\subsection{Results of the multicrystal investigation}

The major practical advantage of the multicrystalline sample (Figs. 1 and 4), which consists of 27 large grains extending over the whole thickness with boundaries nearly perpendicular to the surface, is twofold. First, the entire gauge length can be simulated and subjected to the actual boundary conditions; second, the mesh can be refined easily.

The identification procedure described above has been applied to this sample and to the four constitutive laws A, B, C and D. For optimized parameter values, all these laws lead to global stressstrain responses that are very close to the experimental one. Fig. 5 shows a comparison between optimized simulated reduced $E_{11}$ distributions and the experimental one, for a macroscopic axial strain equal to $5 \%$. The very large spread in the experimental values, from 0.6 to 1.5 times the average value, has to be emphasized first. It reveals a strong heterogeneity of the strain field. Obvi-

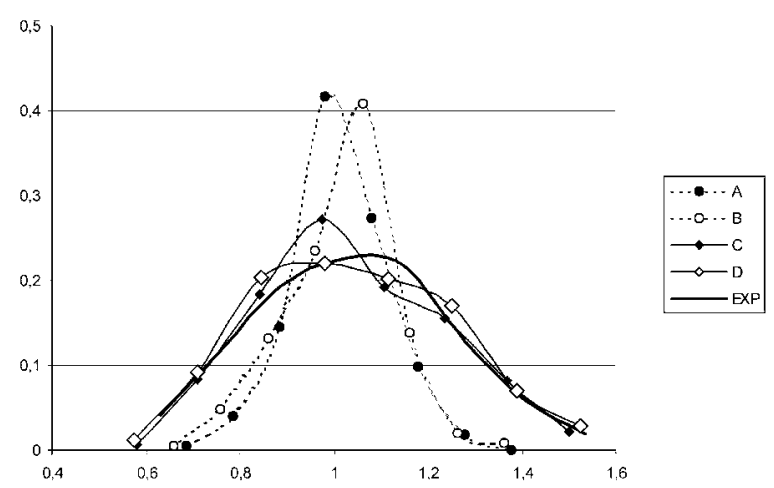

Fig. 5. Experimental and simulated normalized distributions of the $E_{11}$ strain field component after optimization for the four tested constitutive laws (multicrystalline sample; overall axial strain: $5 \%$ ). 
Table 3

Optimal material parameters for the multicrystal

Law

$\mathrm{B} \quad \tau_{0}^{\mathrm{B}}=59 \mathrm{MPa} \quad H_{0}^{\mathrm{B}}=303 \mathrm{MPa} \quad q^{\mathrm{B}}=1.26$

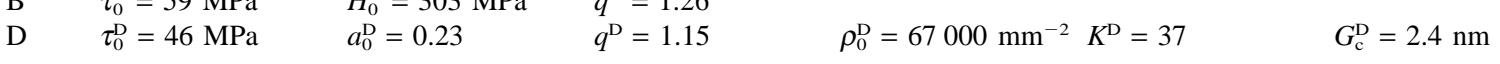

ously, type A and B laws, which are based on a linear hardening description, are unable to capture this important feature. On the other hand, for both linear and nonlinear hardening, strain heterogeneity is favored by latent hardening, with a factor $q$ of the order of 1.2, as expected from the literature (see below).

The optimal values of the material parameters for the multicrystal are given in Table 3 for type $\mathrm{B}$ and $\mathrm{D}$ laws. These values will be discussed in Section 4.

The multicrystalline sample investigated is very convenient for application of the proposed identification procedure. However, it is not representative of normal situations, which are mainly concerned with polycrystalline materials. For this reason, the proposed approach has been validated on an IF-Ti steel polycrystal. The validation is carried out using the $\mathrm{D}$ type law, as the latter yields much better results than the other three.

\section{Validation on a polycrystalline sample}

The chemical composition of the IF-Ti steel is given in Table 4.

This composition is close to that of the ARMCO multicrystal, but is, nevertheless, slightly different. Though the constitutive law D is still likely to be appropriate, the material parameters are not expected to be the same. The polycrystalline sample has a grain size of $20 \mu \mathrm{m}$ and an initial crystallographic texture close to a $<111>$ fiber texture.
For both strain measurements and numerical simulation, an adequate area of the sample has to be defined first. Since only the surface microstructure is to be modeled, it is preferable to select an area large enough to be representative of the whole microstructure. This can be checked by comparing the EBSD measurements to the experimental crystallographic texture derived from X-ray diffraction analysis.

Nevertheless, this surface area cannot be too large, since a sufficient number of points of the deposited microgrids for microextensometry measurements have to be inside the grains in order to capture the intragranular strain heterogeneity. Thus, a compromise has to be found between these contradictory requirements. It leads to an area of $100 \times 100 \mu^{2}$, containing 87 grains and 1600 fiducial grid points with a $2.5 \mu \mathrm{m}$ pitch (Fig. 6).

The $E_{11}$ strain field in this area is shown in Fig. 7 for an overall axial strain of $2.25 \%$. As for the multicrystal, localized strain bands inclined at an angle of roughly $\pm 45^{\circ}$ with respect to the tensile axis can already be observed at this strain value.

The reduced statistical distribution of the measured axial strain field is shown in Fig. 8 for three values of prescribed axial strain, namely $2.25 \%$, $7.5 \%$ and $26.8 \%$. The amplitude of this reduced quantity is quite constant whatever the macroscopic strain. Note that at the lower strain (2.25\%), the distribution looks bimodal rather than Gaussian, in contrast to what is observed at larger strains $(7.5 \%$ and $26.8 \%)$. This evolution could be the consequence of some spreading of the initial

Table 4

Chemical composition of the polycrystalline sample $\left(10^{-3}\right.$ wt.\%)

\begin{tabular}{llllllllllll}
\hline & $\mathrm{C}$ & $\mathrm{Mn}$ & $\mathrm{P}$ & $\mathrm{S}$ & $\mathrm{Al}$ & $\mathrm{Ti}$ & $\mathrm{Si}$ & $\mathrm{Cu}$ & $\mathrm{Cr}$ & $\mathrm{Ni}$ & $\mathrm{Fe}$ \\
\hline $\mathrm{IF}-\mathrm{Ti}$ & 4 & 129 & 7 & 5 & 44 & 108 & 13 & 6 & 18 & 20 & $\mathrm{Bal}$ \\
\hline
\end{tabular}




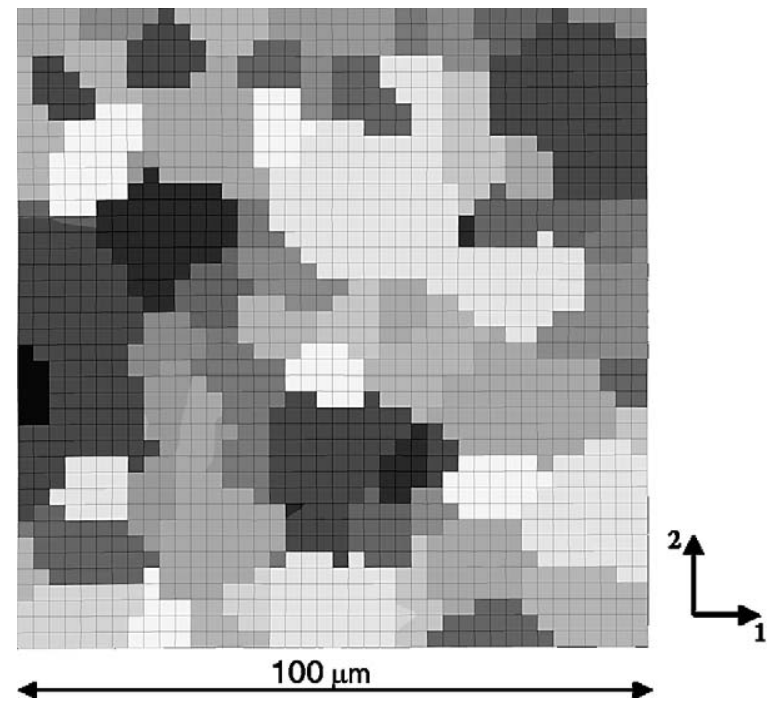

Fig. 6. Selected area for the investigation of the polycrystalline sample.

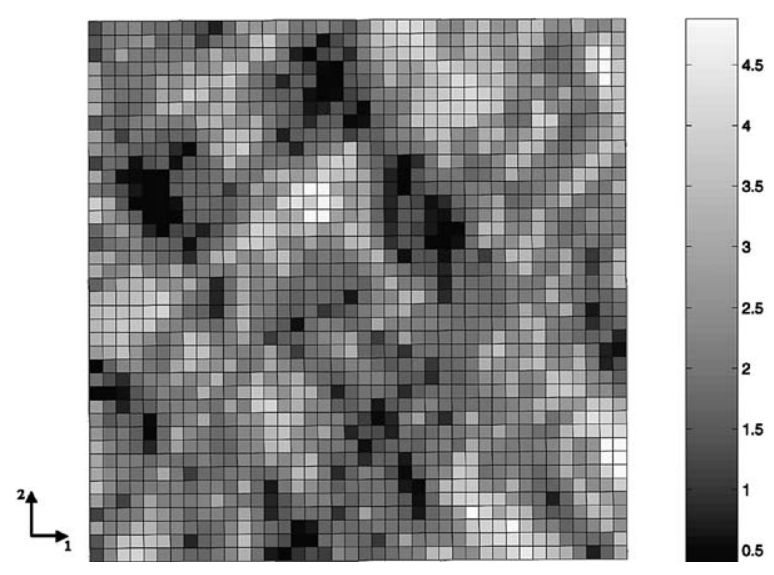

Fig. 7. Experimental $E_{11}$ axial strain field for the polycrystalline sample (overall axial strain: $2.25 \%$ ).

localized strain bands at larger strain, due to work hardening.

An adequate definition of the boundary conditions is needed for the FEM simulation. Although the area under study was large enough to be reasonably representative, applying the macroscopic homogeneous conditions imposed on the whole sample may lead to errors. Since the displacement field is to be measured, it seems more sensible to use the values measured at the border

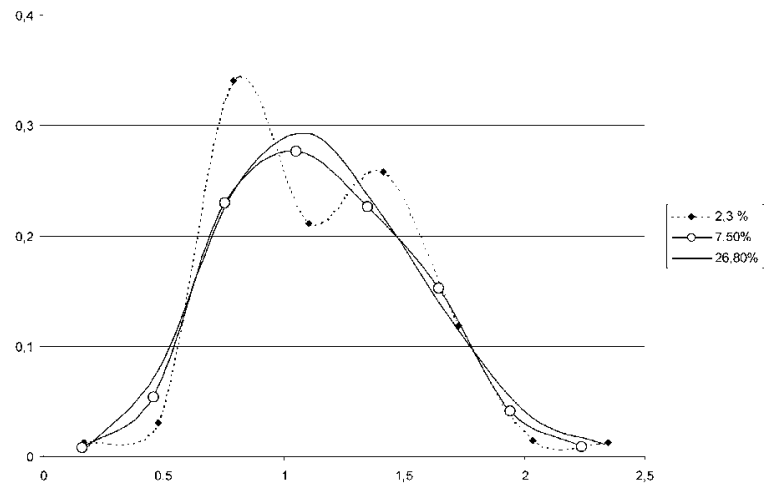

Fig. 8. Statistical distribution of the measured normalized local strain $\left(E_{11}\right)$ values for the polycrystalline sample (macroscopic strain: $2.25 \%, 7.5 \%$ and $26.8 \%$ ).

of this area as boundary conditions [11]. The mesh used consists of one layer of elements (C3D8R) with prescribed in-plane boundary conditions from the experimental data and free surface conditions for the normal direction.

As mentioned above, use is made of constitutive law $\mathrm{D}$, with anisotropic nonlinear hardening. The optimal parameters are identified at a macroscopic strain of $2.25 \%$ (Table 5). As expected, these parameters are not very different from those derived for the ARMCO multicrystal (Table 3). Nevertheless, differences do exist, which deserve discussion (Section 4).

Fig. 9 shows the evolution of the simulated statistical distribution of the reduced local axial strain for several macroscopic strains. The experimental distribution at $7.5 \%$ is also reproduced. The simulated distributions seem to have a Gaussian shape and look more homogeneous than the experimental ones. The shape of the distribution stabilizes beyond strains of $8-9 \%$, that is, later than the experimental values (Fig. 8). However, globally, there is fairly good agreement between experiments and simulations.

It is now possible to analyze the spatial distribution of the local strain field, which was not taken into account in the identification procedure. Fig. 10 represents the computed strain field $E_{11}$ for a macroscopic strain of $2.25 \%$. When compared to the experimental field (Fig. 7), this figure shows very close similarity. In particular, the pattern of localized deformation bands corresponds rather 
Table 5

Optimal material parameters for the polycrystal

\begin{tabular}{|c|c|c|c|c|c|c|}
\hline Polycrystal & $\mathrm{MPa}$ & & & $\mathrm{mm}^{-2}$ & & $\mathrm{~nm}$ \\
\hline D law & $\tau_{0}^{\mathrm{P}}=60$ & $a_{0}^{\mathrm{P}}=0.29$ & $q^{\mathrm{P}}=1.2$ & $\rho_{0}^{\mathrm{P}}=78000$ & $K^{\mathrm{P}}=24$ & $G_{\mathrm{c}}^{\mathrm{P}}=1.3$ \\
\hline
\end{tabular}

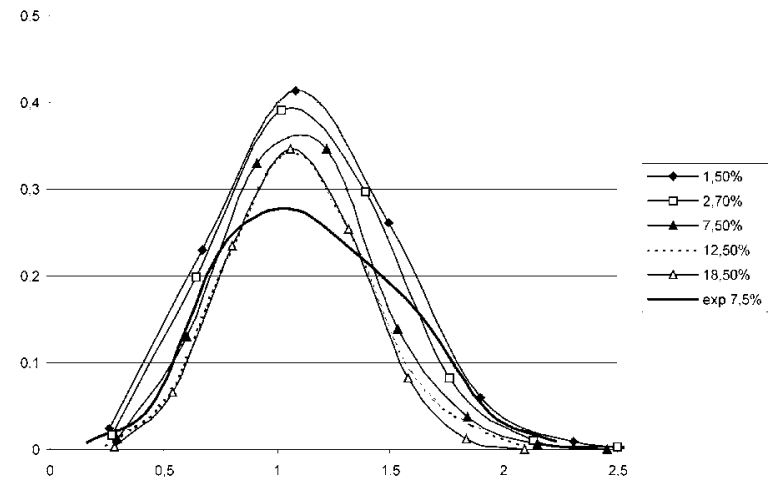

Fig. 9. Experimental (at 7.5\%) and simulated (at 1.5\%, 2.7\%, $7.5 \%, 12.5 \%$ and $18 \%$ ) normalized distributions of the $E_{11}$ strain field component after optimization for the D type constitutive law (polycrystalline sample).

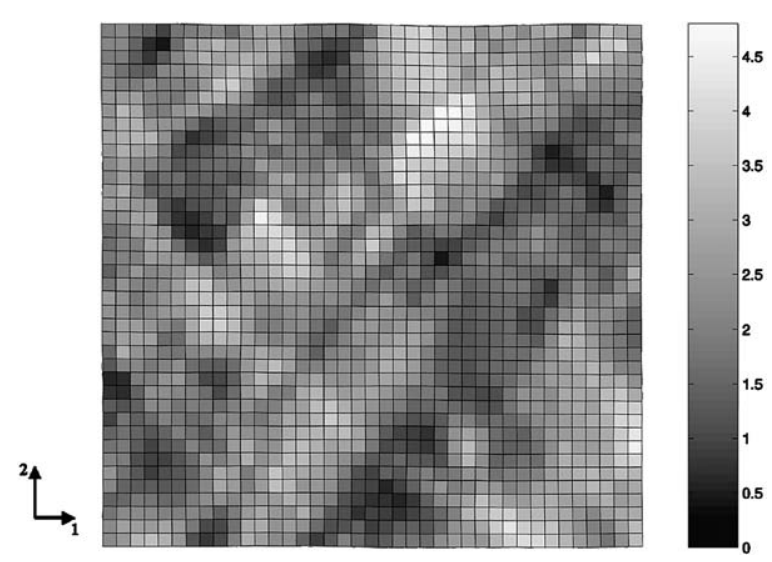

Fig. 10. Computed $E_{11}$ strain field at $2.25 \%$ (polycrystalline sample).

well to the experimental one, except in the upper right corner, which might be due to a specific microstructural environment beneath the surface.

The validation of the present approach can also be made from a more macroscopic point of view, by comparing the evolution of crystallographic tex- tures (Fig. 11) and the macroscopic strain-stress curves (Fig. 12). These two figures show that, at least for the grain lattice orientations, the area investigated is a reasonably representative one. Constitutive law D is able to reproduce both the macroscopic behavior of the studied material and the evolution of its crystallographic texture rather well. However, the macroscopic localization associated with global softening occurs earlier in the simulation than in the experiment. (a)

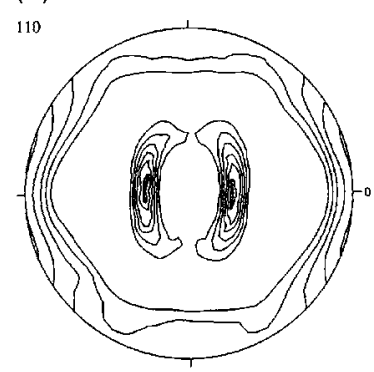

(c)

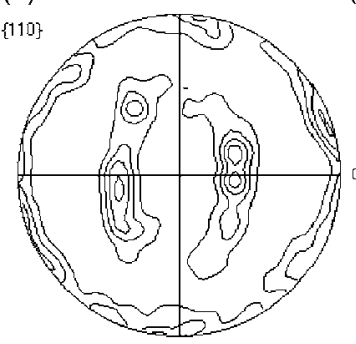

(b)

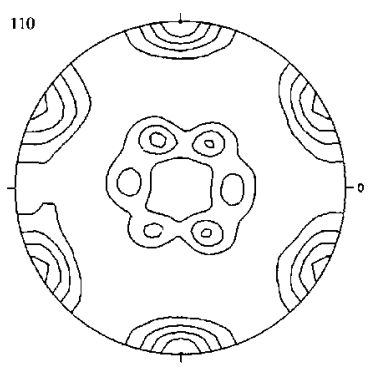

(d)

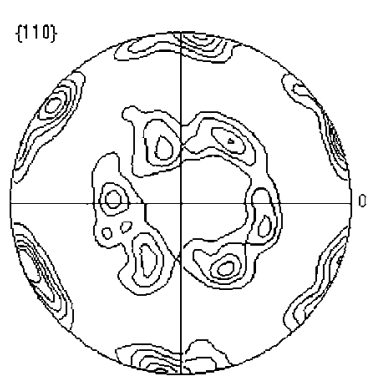

Fig. 11. Comparison of computed and experimental texture evolution: (a) initial X-ray diffraction experimental pole; (b) experimental X-ray diffraction pole figure after a $27 \%$ tensile deformation along the transverse direction; (c) experimental EBSD pole figure on the selected subarea in the initial state; (d) corresponding simulated pole figure after a $27 \%$ deformation along the transverse direction. 


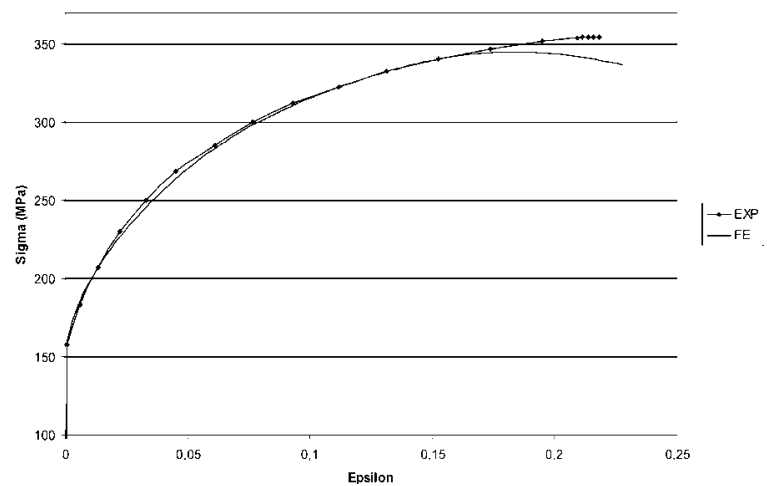

Fig. 12. Experimental (EXP) and simulated (FE) macroscopic strain-stress curves for the polycrystal.

\section{Discussion and conclusion}

The foregoing analysis shows that, from a macroscopic point of view, the crystallographic textures and overall responses obtained from experimental results and simulations are very close to each other quantitatively. The same conclusion holds for the localization pattern of the strain heterogeneity. This good agreement can be due to the fact that the boundary conditions applied to the simulation come from experimental measured displacements. Another simulation with homogeneous boundary conditions is shown in Fig. 13. We observe that the strain localization pattern is now quite different and differs significantly from the

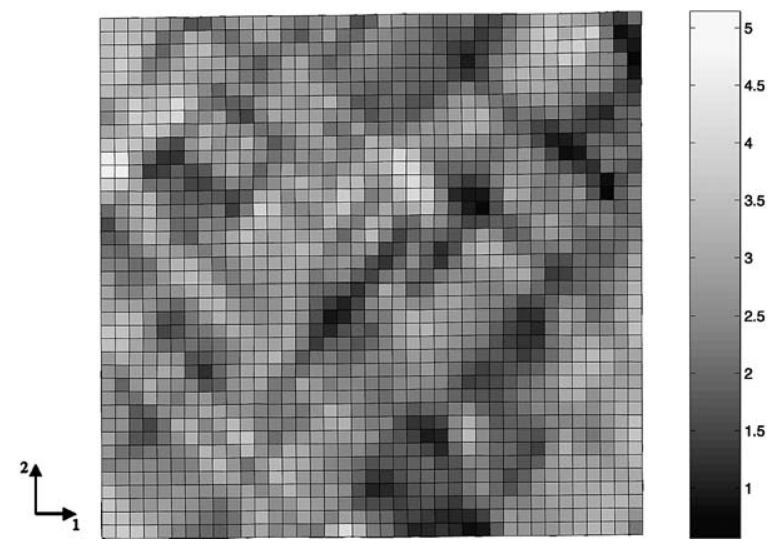

Fig. 13. Component $E_{11}$ of the strain field for the polycrystal obtained by finite element computation with homogeneous boundary conditions. experimental one. It can be understood that the use of experimental boundary conditions allows us not only to take into account the actual in-plane heterogeneous loading but also, in a reasonable way, to integrate information concerning the subsurface microstructure of the sample.

Secondly, we observe that constitutive equations of types A and B can hardly reproduce more than the overall response for a given simple loading path because of their phenomenological character. For the materials investigated, latent hardening seems to affect the amplitude of the local strain heterogeneity in a significant manner. Constitutive law $\mathrm{D}$ is found to be realistic enough to reproduce the main characteristics of the experimental local responses. It is then interesting to compare the values of the associated optimal material parameters with those found in the literature. The value of parameter $G_{\mathrm{c}}$, which characterizes a distance associated with the annihilation of dislocation dipoles [32,33], is assumed to be a few Burgers vectors. Here, the optimal value for $G_{\mathrm{c}}$ is consistently found to be of the order of 5-10b. As for the initial mean free-path parameter $L$, which is related to $K$ and $\rho_{0}$ by the relation

$$
L^{s}=\frac{K}{\sqrt{\sum_{u \neq s} \rho_{0}}}
$$

its value is expected to be typically a few tens of micrometers. According to our results, it would be $30 \mu \mathrm{m}$ for the multicrystal and $18 \mu \mathrm{m}$ for the polycrystal. This last value is consistent with the grain size of the polycystal $(20 \mu \mathrm{m})$. Moreover, we find, for the multicrystal, a mean free-path twice as large as the one found for the polycrystal, which follows the right direction. The description of the hardening used in this paper is an extension of the Mecking-Kocks evolutionary law, with a lattice friction parameter and a term due to the interaction of dislocations. The average value of the interaction coefficient $\left(\sqrt{\left(1 / N_{\mathrm{sys}}\right) \sum_{u} a^{(s)(u)}}\right)$ is 0.5 , which is consistent with values mentioned in the literature [37]. The conclusion is the same for the latent hardening parameters [30,38,39].

Concerning these parameters, the literature shows that, as long as we are interested in the 
Table 6

Initial and final optimized values for the parameters of the D law (polycrystal)

\begin{tabular}{lllllll}
\hline & $\tau_{0}^{\mathrm{P}}(\mathrm{MPa})$ & $a_{0}^{\mathrm{P}}$ & $q^{\mathrm{P}}$ & $\rho_{0}^{\mathrm{P}}\left(\mathrm{mm}^{-2}\right)$ & $K^{\mathrm{P}}$ & $G_{\mathrm{c}}^{\mathrm{P}}(\mathrm{nm})$ \\
\hline $\mathrm{Ai}$ & 46 & 0.23 & 1.15 & 67000 & 37 & 2.4 \\
$\mathrm{Af}$ & 60 & 0.29 & 1.2 & 78000 & 24 & 1.3 \\
$\mathrm{Bi}$ & 61 & 0.5 & 1.42 & 30000 & 10 & 5 \\
$\mathrm{Bf}$ & 64.4 & 0.26 & 1.19 & 72000 & 21.5 & 1.2 \\
\hline
\end{tabular}

macroscopic response to a monotonic uniaxial loading, it is not necessary to take into account the latent hardening parameter [40]. On the contrary, if one wishes to predict the mechanical behavior of a material from its response to a sequential loading [22], the interaction matrix has to be nonuniform, in order to account for the different interactions that are observed experimentally [38]. These results show a direct relation between the local heterogeneity and the hardening mechanisms. Thus, it is clear that only a matrix containing more than two different components is able to predict the local strain heterogeneity of the polycrystal fully. However, the constitutive law used in this paper (with only two terms) has been found to describe the heterogeneity of the multicrystal more satisfactorily than that of the polycrystal. From a physical point of view, the major difference between these two microstructures lies in the influence of the grain boundaries on the local strain distribution. In the multicrystal case, only a few measured points are affected directly by the grain boundaries, and the activation of a new event inside a grain, for instance as a new activated slip system, does not affect the adjacent grains very much. Most of the measured points undergo a quasi-monotonic loading path. In contrast, in the case of a polycrystalline material with small grain size, all the points are affected by the response of the neighboring grains and the local loading path is certainly not monotonous.

One of the major potential errors of this inverse identification procedure originates in the fact that a local optimum can be found, instead of the global one that is looked for. In order to check this point, we have modified the initial values of the set of parameters. Table 6 shows the results obtained for the polycrystalline material and hardening law D.
Two sets of initial parameters ( $\mathrm{Ai}, \mathrm{Bi})$ have been used; Af and $\mathrm{Bf}$ are the parameters obtained after identification. The resulting relative difference between each optimized parameter of the two sets is less than $10 \%$.

Similarly, a refinement of the mesh by a factor of 2.5 has been realized and is found not to modify the simulation results significantly.

Finally, we stress the fact that, at this stage, the proposed identification procedure based on the comparison between experimental and simulated results has only taken into account a few data, namely the local strain distribution and the macroscopic response. From a mathematical point of view, more efficient use could have been made of all the displacement fields in the resolution of the inverse problem. Such an investigation is now in progress. Nevertheless, it can already be concluded that a simplified identification procedure coupled with an experimental investigation performed on a polycrystal material is able to lead to satisfactory material parameter values for the physical constitutive laws. No tests on single crystals are needed. Consequently, these material parameters can be used in turn more confidently at the crystal level in order to better predict the overall polycrystal response through adequate upscaling models.

\section{Acknowledgements}

D. Caldemaison and M.L. Giorgi are gratefully acknowledged for their help during experiments. The experimental strain fields were computed using the program "correlmanuv" kindly provided by M. Bornert and P. Doumalin. 


\section{References}

[1] Masson R, Zaoui A. J Mech Phys Solids 1999;47:1543.

[2] Gilormini P, Nebozhyn M, Ponte Castañeda P. Acta Mater 2001;49:329.

[3] Bornert M, Masson R, Ponte Castañeda P, Zaoui A. J Mech Phys Solids 2001;49:2737.

[4] Taylor GI. J Inst Met 1938;61:307.

[5] Hill R. J Mech Phys Solids 1965;13:89.

[6] Hutchinson JW. Proc R Soc London A 1976;348:101.

[7] Lineau C, Rey C, Viaris P. Mater Sci Eng A 1997;234236:853.

[8] Doumalin P. PhD thesis, Ecole Polytechnique, Palaiseau, France, 2000.

[9] Perie JN, Calloch S, Cluzel C, Hild F. Exp Mech 2002;42:318.

[10] Roux S, Hild F, Berthaud Y. Appl Opt 2002;41:108.

[11] Soppa E, Doumalin P, Binkele P, Wiesendanger T, Bornert M, Schmauder S. Comput Mater Sci 2001;21:261.

[12] Harren SV, Asaro RJ. J Mech Phys Solids 1989;37:191.

[13] Kalidindi SR, Bronkhorst CA, Anand L. J Mech Phys Solids 1992;40:5376.

[14] Becker R. Acta Metall 1991;39:1211.

[15] Teodosiu C, Raphanel JL, Tabourot L. In: Teodosiu C, Raphanel JL, Sidoroff F, editors. Large plastic deformations. Rotterdam: Balkema; 1993. p. 15-3.

[16] Harder J. Int J Plast 1998;15:605.

[17] Becker R, Panchanadeeswaran S. Acta Metall 1995;43:2701.

[18] Delaire F, Raphanel JL, Rey C. Acta Metall 2000;48:1075.

[19] Hoc T, Rey C. Scr Mater 2000;42:1053.

[20] Tabourot L, Fivel M, Rauch E. Mater Sci Eng A 1997;234-236:639.
[21] Arizmendi D, Raphanel JL. Mater Res Soc Boston 1999;578:27.

[22] Hoc T, Forest S. Int J Plast 2001;17:65.

[23] Claire D, Hild F, Roux S. C R Mec 2002;330:729.

[24] Gélébart L. PhD thesis, Ecole Polytechnique, Palaiseau, France, 2002.

[25] Allais L, Bornert M, Bretheau T, Caldemaison D. Acta Metall Mater 1994;42:3865.

[26] Smelser RE, Becker R. In: ABAQUS user subroutines for material modeling, 1989.

[27] ABAQUS user's manual, Hibbit, version 5.8. Providence: Karlsson \& Sorensen.

[28] Peirce D, Asaro RJ, Needlman A. Acta Metall 1983;31:1951.

[29] Chin GY. Metall Trans A 1972;3:2213.

[30] Keh AS, Nakada Y. Can J Phys 1967;45:1101.

[31] Hoc T, Rey C, Raphanel JL. Acta Mater 2001;49:1835.

[32] Essman U, Mughrabi H. Philos Mag A 1979;40:731.

[33] Mecking H, Kocks UF. Acta Metall 1981;29:1875.

[34] Tang M, Devincre B, Kubin L. Model Simul Mater Sci Eng 1999;7:893.

[35] Rauch E. In: Proceedings of the ISPMA6. Key engineering materials, vol. 97-98. 1994:371.

[36] Pilvin P. Inelastic behaviour of solids. In: Oytana C, editor. Proceedings of the International Seminar Mécamat, Besançon. 1988:155.

[37] Keh AS, Weissmann S. In: Thomas G, Washburn J, editors. Electron microscopy and strength of crystals. New York: Interscience. 1963:231.

[38] Franciosi P. Acta Metall 1985;33:1601.

[39] Franciosi P. PhD thesis, Université Paris XIII, Villetaneuse, France, 1984.

[40] Rauch EF, Thuillier S. Mater Sci Eng A 1993;164:255. 